\title{
СОВРЕМЕННЫЙ ВЗГЛЯД НА ЗАМЕСТИТЕЛЬНУЮ ГОРМОНАЛЬНУЮ ТЕРАПИЮ
}

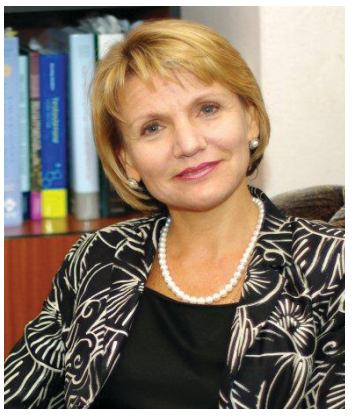

Т.Ф. ТАТАРЧУК

д.мед.н., просрессор, член-кор. НАМН Украины, заместитель директора по научной работе, заведующая отделением эндокринной гинекологии Института педиатрии, акушерства и гинекологии НАМН Украины

\section{О.А. ЕФИМЕНКО}

к.мед.н., старший научный сотрудник отделения эндокринной гинекологии Института педиатрии, акушерства и гинекологии НАМН Украины $\prod_{\text {or }}^{\text {pos }}$ роблема качества жизни женщин в период пери- и постменопаузы становится все более острой и с годами не утрачивает своей актуальности. Это связано прежде всего с увеличением продолжительности жизни, так называемым старением населения планеты и продлением возраста трудоспособности, что приобретает не только медицинское, но и социально-экономическое значение как в нашей стране, так и в других развитых странах.

Развитие тяжелого климактерического синдрома в менопаузе, сопровождающегося значительными нарушениями в различных органах и системах, все так же остается междисциплинарной проблемой с множеством дискутабельных и часто непоследовательных и необоснованных подходов к лечению этой категории пациенток.

Несмотря на активную пропаганду здорового образа жизни от юности до старости и существование целого ряда профилактических и геропротекторных программ, направленных на сохранение здоровья женщин, именно заместительная гормональная терапия (ЗГТ) остается золотым стандартом и главным патогенетически обоснованным медикаментозным методом лечения климактерических нарушений.

Поэтому вопросы о пользе и рисках применения ЗГТ в современном мире все больше привлекают внимание ученых и ведущих специалистов, занимающихся проблемой здоровья женщин. Так, с 8-го по 11 июня 2011 г. в Риме состоялся 13-й Всемирный конгресс по менопаузе. На нем была представлена обновленная версия рекомендаций (International Menopause Society, IMS) Международного общества менопаузы по применению ЗГТ в постменопаузальный период и стратегий сохранения здоровья в среднем возрасте. В данной статье хотелось бы остановиться на основополагающих главах этого документа, что позволит не только расширить кругозор специалистов, занимающихся вопросами здоровья женщин в менопаузе в нашей стране, но и, как говорится, быть на гребне волны, используя обобщенный самый современный опыт ведущих европейских ученых.

Обновленная версия рекомендаций IMS от 2011 г. публикуется во время, когда атмоссрера вокруг вопроса о применении ЗГТ в постменопаузальный период стала более взвешенной и благоприятной. Маятник качнулся обратно из наиболее отрицательной точки после получения подробных результатов исследования Women's Health Initiative (WHI), показавших важность возраста для начала терапии и хороший профиль безопасности ЗГТ в случае применения у женщин моложе 60 лет. И именно на возраст и раннее начало применения ЗГТ обращается внимание в последних рекомендациях ведущих специалистов.

Для повышения качества медицинского сопровождения пациенток в возрасте после 40 лет хотелось бы остановиться на ключевых моментах этого документа. В рекомендациях IMS под термином «заместительная гормональная терапия» подразумеваются виды лечения с использованием эстрогенов, прогестагенов, комбинаций препаратов, андрогенов и тиболона.

В разделе «Основные принципы» рекомендаций IMS (2011) отмечено следующее:

( Решение о назначении ЗГТ должно быть частью стратегии, включающей рекомендации в отношении таких аспектов образа жизни, как питание, фризические упражнения, отказ от курения, безопасный уровень потребления алкоголя и др.

3 ЗГТ следует назначать индивидуально и подбирать с учетом симптомов и необходимости профилактики, а также индивидуального и семейного анамнеза, результатов соответствующих исследований, предпочтений и ожиданий женщины. Риски и польза ЗГТ отличаются у женщин в период перехода к менопаузе и у лиц более старшего возраста.

Женщины, у которых спонтанная или ятрогенная менопауза наступает в возрасте до 45 и особенно до 40 лет, имеют повышенный риск сердечно-сосудистых заболеваний (ССЗ) и остеопороза, а также более высокий риск развития аффрективных расстройств и деменции. ЗГТ может смягчать симптомы и сохранить плотность костной ткани, и ее применение рекомендуется продолжать по меньшей мере до достижения среднего возраста возникновения менопаузы.

(3) Не следует проводить ЗГТ без четких показаний к ее применению, т.е. при отсутствии значительных симптомов или фризических проявлений недостаточности эстрогенов.

Женщинам, принимающим ЗГТ, следует как минимум один раз в год проходить обследование у гинеколога, включая обсуждение образа жизни и разработку стратегии по предотвращению или смягчению течения хронических заболеваний, а также консультации у смежных специалистов при необходимости. Показаний к более частому маммографическому обследованию или скрининговому анализу мазка из шейки матки в настоящее время нет. 
П Причин для обязательных ограничений продолжительности ЗГТ не имеется. Решение о продолжении или прекращении терапии должны принимать хорошо инорормированная пациентка и ее врач, в зависимости от целей лечения и объективной оценки пользы и риска на текущий момент.

Дозу препарата следует титровать до наименьшей эффрективной. Более низкие дозы ЗГТ, чем те, которые использовались в практике ранее, могут в достаточной мере уменьшать симптомы и поддерживать качество жизни у многих женщин.

- Прогестерон следует добавлять к системному эстрогену при лечении всех пациенток с сохраненной маткой для предупреждения развития гиперплазии и рака эндометрия.

В целом, говоря о положительном действии ЗГТ, следует подчеркнуть ее доказанное положительное влияние при эстрогендесицитных постменопаузальных нарушениях, а именно:

3 ЗГТ остается наиболее эффрективным средством при вазомоторных симптомах и атрофрии органов мочеполовой системы. На фоне ЗГТ также возможно улучшение других обусловленных менопаузой нарушений, таких как боль в суставах и мышцах, перепады настроения, нарушения сна, половая диссункция (в т.ч. снижение либидо). Также отмечено ее положительное влияние на качество жизни.

\section{БЛАГОПРИЯТНЫЕ ЭФФЕКТЫ ЗГТ}

\section{Постменопаузальный остеопороз}

ЗГТ эфффективно предотвращает потерю костной ткани, обусловленную наступлением менопаузы, и снижает частоту всех переломов, связанных с развитием остеопороза, в Т.ч. позвонков и бедра, даже у женщин с невысоким их риском. Исходя из данных об эффективности, стоимости и безопасности, ЗГТ может рассматриваться как один из видов терапии первого ряда для предотвращения и лечения остеопороза у женщин в постменопаузе младше 60 лет с высоким риском переломов.

На сегодняшний день доказано, что защитное действие ЗГТ на минеральную плотность костной ткани после прекращения терапии снижается с непредсказуемой скоростью, хотя некоторая степень защиты может сохраняться и после прекращения ЗГТ.

\section{Сердечно-сосудистые заболевания}

ССЗ являются основной причиной заболеваемости и смертности женщин в период менопаузы. Основными первичными профрилактическими мерами (помимо отказа от курения и контроля за питанием) являются снижение массы тела, нормализация артериального давления (АД), регулярные фризические упражнения, контроль уровня сахара и липидов. ЗГТ обладает потенциалом снижения риска развития ССЗ благодаря положительному действию на функцию сосудов, уровень холестерина, метаболизм глюкозы и АД.
Имеются доказательства того, что терапия эстрогенами может оказывать кардиопротекторное действие, если ее начать в пре- или перименопаузе и продолжать в течение длительного периода времени (концепция «окна терапевтических возможностей»). ЗГТ снижает риск развития сахарного диабета и СС3, влияя на липидный профиль и метаболический синдром.

У женщин в возрасте младше 60 лет, у которых симптомы менопаузы появились недавно и не выявлено признаков ССЗ, инициация ЗГТ не вызывает ранних неблагоприятных последствий и может снизить заболеваемость и смертность от ишемической болезни сердца (ИБС). Решение о продолжении ЗГТ у таких женщин после 60 лет должно быть принято на основании общей оценки соотношения польза/риск.

Инициация ЗГТ у лиц старше 60 лет, а также у пациенток с длительностью менопаузы более 10 лет может обусловливать повышение риска развития кардиоваскулярных заболеваний преимущественно в первые 2 года применения. Поэтому проводить ЗГТ у женщин старше 60 лет исключительно с целью первичной профрилактики ИБС не рекомендуется.

\section{Другие благоприятные эфффекты}

Системная ЗГТ и эстрогены для местного применения обладают способностью предотвращать атрофические изменения мочеполового тракта, обусловленные недостаточностью эстрогенов, и поддерживать слизистую влагалища в соответствующем возрасту здоровом состоянии. ЗГТ также благоприятно влияет на состояние соединительной ткани, кожи, суставов и межпозвоночных дисков. Применение в качестве таковой комбинации конъюгированных конских эстрогенов и медроксипрогестерона ацетата в течение более 4 лет может снизить риск развития рака толстой кишки. ЗГТ, инициированная в период перименопаузы или применяемая у женщин более молодого возраста в постменопаузе, ассоциируется со снижением риска развития болезни Альцгеймера.

\section{ВОЗМОЖНЫЕ СЕРЬЕЗНЫЕ НЕЖЕЛАТЕЛЬНЫЕ ЭФФЕКТЫ ЗГТ}

Исследования рисков, связанных с применением гормонов в постменопаузальном периоде, фокусировались преимущественно на раке молочной железы (РМЖ) и эндометрия, венозной тромбоэмболии (легочной эмболии или тромбозе глубоких вен), инсульте и коронарных заболеваниях.

\section{Рак молочной железь}

Частота РМЖ в разных странах варьирует. Степень связи между применением ЗГТ в постменопаузальном периоде и развитием РМЖ остается спорной.

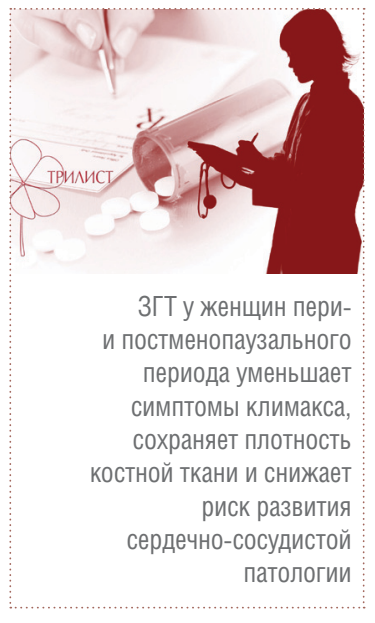

ЗГТ у женщин перистменопаузального ериода уменьшает симптомы климакса, раняет плотность риск развития патологии 


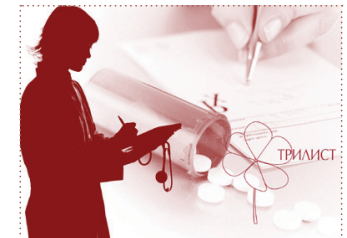

Женщин следует предупредить, что возможное повышение риска РМЖ, связанное с ЗГТ, невелико и является более низким, чем риск развития РМЖ, обусловленный обычными фракторами, такими как ожирение и потребление алкоголя
Женщине необходимо объяснить, что возможное повышение риска развития РМЖ, связанное с ЗГТ, невелико (менее 0,1\% в год или < 1,0 случая на 1000 женщин за год применения) и меньше, чем повышение риска, обусловленного обычными факторами, такими как ожирение и потребление алкоголя. Результаты рандомизированного контролируемого исследования WHI не указывают на повышение риска развития РМЖ в течение 5-7 лет с начала лечения у пациенток, впервые прибегнувших к ЗГТ. Однако большинство участниц исследования WHI имели избыточную массу тела или ожирение, что могло повлиять на исходный уровень риска развития РМЖ.

Женщин следует успокоить, что возможное повышение риска РМЖ, связанное с ЗГТ, невелико и меньше, чем повышенный риск, обусловленный обычными фракторами образа жизни, такими как ожирение и потребление алкоголя.

\section{Рак эндометрия}

Применение эстрогенов в виде монотерапии вызывает дозозависимую стимуляцию эндометрия, поэтому пациенткам с сохраненной маткой следует дополнительно принимать прогестагены для противодействия этому эффекту.

При длительном применении комбинации эстрогенов с прогестагенами отмечена более низкая частота возникновения гиперплазии и рака эндометрия, чем в обычной популяции.

Ряд преимуществ может наблюдаться при внутриматочном применении терапии. Схемы с использованием низких и ультранизких доз эстрогена и прогестагена вызывают меньшую стимуляцию эндометрия и меньше кровотечений.

\section{Тромбоэмболия и сердечно-сосудистые} события

Риск серьезных венозных тромбоэмболических осложнений, связанный с применением ЗГТ, повышается с возрастом (хотя минимален у женщин группы низкого риска до 60 лет), а также при наличии ожирения и тромбофилии. Применение трансдермальных препаратов эстрогенов поможет избежать риска, связанного с препаратами ЗГТ для приема внутрь, благодаря отсутствию первичного печеночного метаболизма. Тип прогестагена также может влиять на риск развития тромбоэмболических событий.

Риск развития инсульта коррелирует с возрастом - у лиц моложе 60 лет он возникает редко. ЗГТ может дополнительно повышать этот риск, особенно после 60 лет. Применение трансдермальных низкодозированных препаратов не повышает риск инсульта. Данные о безопасности, полученные в исследованиях с применением низких и ультранизких доз эстрогенов и прогестагенов, обнадеживающие и свидетельствуют о меньшем числе нежелательных явлений

\section{АЛЬТЕРНАТИВНЫЕ МЕТОДЫ ЛЕЧЕНИЯ СИМПТОМОВ МЕНОПАУЗЫ}

Эффрективность и безопасность альтернативных препаратов не установлены и требуют проведения дальнейших исследований.

По данным краткосрочных испытаний, селективные ингибиторы обратного захвата серотонина и норадреналина, а также габапентин эфрфективно уменьшают вазомоторные симптомы.

Медицинские или научные обоснования для назначения незарегистрированных «биоидентичных гормонов» отсутствуют. Такие «индивидуализированные» гормональные препараты не изучались должным образом в рамках исследований, и риски, связанные с их применением, также неизвестны.

\section{ВЫводы}

ЗГТ в постменопаузальном периоде является частью общей стратегии медицинского наблюдения этой категории пациенток. Необходимо обязательно оценить пользу и риски ее применения, которые могут значительно отличаться в каждом отдельном случае. Важно, что проведенные в последнее десятилетие исследования помогли установить, что риски можно свести к минимуму, а пользу - к максимуму, выбрав оптимальную схему лечения в оптимальное время.

Безопасность ЗГТ значительным образом зависит от возраста. Здоровые женщины моложе 60 лет не должны излишне беспокоиться о профиле безопасности ЗГТ. Результаты недавних исследований и повторный анализ результатов прошлых исследований с учетом возраста женщин показали, что в большинстве случаев потенциальные благоприятные эффректы ЗГТ многочисленны, а риски, наоборот, незначительны, если терапия начата через несколько лет после начала менопаузы.

Что же касается длительности применения ЗГТ, то женщины могут получать ее до тех пор, пока она оказывает благоприятное действие на симптомы. При этом пациентки должны быть осведомлены о рисках, связанных с их схемой лечения, и о возможных рисках, связанных с личными обстоятельствами. Они могут пытаться прекратить лечение каждые несколько лет, но у некоторых из них симптомы менопаузы могут продолжаться многие годы, и их все же необходимо лечить наименьшими эффрективными дозами препаратов.

Здоровые женщины моложе 60 лет не должны излишне беспокоиться о профиле безопасности $3 Г T$

\section{ОСНОВНЫЕ ПОЛОЖЕНИЯ РЕКОМЕНДАЦИЙ Физическая активность в период менопаузы}

9 Регулярная фризическая активность снижает уровень кардиоваскулярной и общей смертности. 
У У сризически активных женщин отмечаются лучшее состояние обмена веществ, координации, мышечного тонуca, когнитивных функций и качества жизни. Кардиальные события, инсульты, переломы, РМЖ и рак толстой кишки встречаются значительно реже.

П Польза от фризических упражнений существенно превышает возможные неблагоприятные последствия - чем больше, тем лучше. Однако излишняя активность может причинить вред.

\section{Здоровый образ жизни}

3 Ожирением (индекс массы тела > 30 кг/м²) страдает более $20 \%$ женской популяции во многих странах мира. Значимость проблемы ожирения все больше возрастает в определенных социальных слоях общества, а также среди детей.

5 Снижение веса всего на 5-10\% является важным для улучшения состояний, связанных с синдромом инсулинорезистентности.

Ос Основные принципы здорового питания: несколько порций фрруктов и овощей в день, потребление пищевой клетчатки, рыба два раза в неделю, сокращение потребления жиров. Ограничение потребления соли, суточная порция алкоголя не должна превышать 30 г для мужчин и 20 г для женщин.

3 Отказ от курения.

Сзменение образа жизни, включающее социализацию, фризическую и умственную активность.

\section{Урогинекология}

3 Такие симптомы, как сухость и болезненность влагалища, диспареуния, частое мочеиспускание, никтурия, частые позывы к мочеиспусканию, крайне распространены у женщин в постменопаузальный период. Распространенность недержания мочи с возрастом увеличивается. В целом 25\% женщин сообщают о недержании мочи, из которых 7\% считают его значительным; 50\% женщин жалуются на недержание мочи при стрессах, $11 \%$ - на императивное недержание и $36 \%$ - на смешанное недержание.

与 Урогенитальные симптомы хорошо купируются эстрогенами. Часто требуется длительная терапия, поскольку симптомы могут возобновляться после прекращения лечения. Системные риски при применении местных эстрогенов с низким потенциалом и в низкой дозе не определены.

У У лиц в постменопаузальном периоде с гиперактивностью мочевого пузыря после изменения образа жизни и ретренинга мочевого пузыря терапией первой линии являются антимускариновые препараты в сочетании с местными эстрогенами.

Для всех женщин, жалующихся на стрессовое недержание мочи, в первую очередь будет полезной тренировка мышц тазового дна. Антидепрессант дулоксетин может действовать синергично с консервативной терапией. Однако многим женщинам требуется оперативное лечение, и наиболее часто с этой целью применяются ретролобковая и трансобтураторная петли.

3 В настоящее время системная терапия эстрогенами не рекомендуется женщинам со стрессовым недержанием мочи.

\section{Остеопороз}

\section{Общие рекомендации}

Лечение

( Целью лечения при остеопорозе является предотвращение переломов. При выборе терапии следует исходить из соотношения эффективности, рисков и стоимости.
Порог вмешательства для назначения терапии может основываться на 10-летней вероятности переломов и будет специфическим в каждой отдельной стране.

( Соотношение стоимость/эфффективность терапии с целью профилактики переломов при остеопорозе более выгодно применять у женщин с повышенным риском переломов. Порог относительного риска переломов является специфичным в зависимости от системы здравоохранения.

\section{Гормональная терапия}

3ГТ эфффективна в профилактике потери костной ткани, связанной с наступлением менопаузы или вторичной аменореей.

3 ЗГТ снижает частоту всех переломов, связанных с остеопорозом, в т.ч. переломов позвонков и бедра, даже в популяции женщин без высокого риска переломов.

3ГТ является одной из стратегий первого выбора для лечения женщин в постменопаузе в возрастной группе 50-60 лет со значительным риском переломов.

3 Защитное действие ЗГТ на минеральную плотность костной ткани утрачивается с непредсказуемой скоростью после прекращения терапии. Хотя некоторая степень защиты от переломов может сохраняться после прекращения ЗГТ, пациенткам группы высокого риска развития переломов может понадобиться дополнительное лечение препаратами с доказанными свойствами сохранения костной ткани.

5 При продолжении ЗГТ у женщин после 60 лет единственно с целью профилактики переломов следует учитывать возможные побочные эффекты, которые могут развиться при применении определенной дозы и определенной схемы ЗГТ по сравнению с другими проверенными методами лечения.

5 Начинать ЗГТ после 60 лет только с целью предотвращения переломов не рекомендуется.

\section{Негормональная терапия}

Кальций и витамин D

Рекомендуемое ежедневное потребление кальция с пищей для женщин в постменопаузе составляет 1000-1200 мг.

5 Добавки, содержащие кальций в количестве, превышающем рекомендуемое потребление с пищей (общее потребление), могут неблагоприятно влиять на сердечнососудистую систему.

( Рекомендуемое потребление витамина D с пищей в постменопаузальном периоде составляет 800-1000 МЕд.

Бисфосфонаты

(5исфосфонаты являются мощными ингибиторами резорбции костей и снижают скорость ремоделирования кости с доказанной эфффективностью в профилактике переломов позвонков и бедра.

5. Бисфоссронаты оказывают благоприятное действие при некоторых онкозаболеваниях и препятствуют метастазированию РМЖ в костную ткань.

\section{Кожа, хрящевая ткань и другие соединительные ткани}

\section{Кожа, сонная артерия и межпозвоночные диски}

5 Эстрогены благоприятно влияют на метаболизм соединительной ткани во всем организме.

Ђ После менопаузы отмечается потеря соединительной ткани в дерме кожи, темпы которой при применении эстрогенов в некоторых случаях значительно замедляются. 


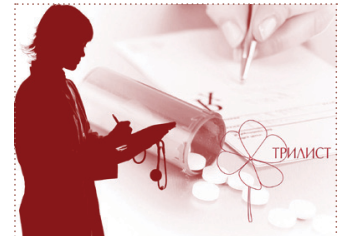

Женщинам, принимающим ЗГТ, следует не реже

одного раза в год проходить обследование, включающее:

- физикальное обследование

- пересмотр индивидуального

медицинского и семейного

анамнеза;

- лабораторные

и визуализирующие методы исследования;

- обсуждение образа жизни;

- врачебные рекомендации

по профилактике или

лечению хронических

заболеваний
(Подобные изменения соединительной ткани отмечаются и в стенках артерий.

s После наступления менопаузы межпозвоночные диски истончаются, и терапия эстрогенами может предупредить этот процесс.

\section{Суставные хрящи и менопауза}

3 Выраженное преобладание полиартикулярного остеоартрита и в частности выраженное повышение частоты развития остеоартрита у женщин после менопаузы свидетельствует о важности женских половых гормонов для гомеостаза хрящей

5 Своевременное начало терапии эстрогенами/селективными модуляторами эстрогенных рецепторов может предотвратить потерю костной и хрящевой ткани, сопутствующую менопаузе, посредством прямых и косвенных механизмов.

\section{Сердечно-сосудистая система}

5 Менопауза может считаться фрактором риска развития ИБС у женщин в связи с потенциальным влиянием угасания фрункции яичников на функцию сердечно-сосудистой системы, АД и различные метаболические параметры (толерантность к глюкозе, профиль липидов).

Стратегия профилактики должна быть направлена на снижение АД, контроль веса и метаболизма глюкозы. Стенокардия у пациенток чаще протекает без обструкции коронарных артерий, но в случае развития инфраркта прогноз у женщин значительно хуже, чем у мужчин.

Гормональная терапия в постменопаузе и ИБС

5 Большая часть результатов доклинических и обсервационных исследований подтверждает потенциальную пользу ЗГТ в снижении риска развития ИБС. Эстрогены благоприятно влияют на различные метаболические факторы риска ИБС. Установлено, что снижение риска развития впервые выявленного сахарного диабета связано с применением ЗГТ.

- Результаты масштабных рандомизированных и обсервационных исследований свидетельствуют о важности возраста для начала применения гормональных препаратов. Благоприятное действие на коронарные сосуды отмечалось только у тех лиц, менопауза у которых наступила менее 10 лет назад.

3 ЗГТ не рекомендуется начинать у пожилых женщин с ИБС.

\section{Влияние ЗГТ на инсульт}

5 Повышенный абсолютный риск при ЗГТ будет ниже у женщин в возрасте до 60 лет, поскольку частота инсульта ниже в более молодой возрастной группе. После наступления менопаузы не отмечается значительных изменений относительного риска инсульта в зависимости от возраста или времени с момента наступления менопаузы.
По данным единичного, должным образом спланированного клинического исследования с участием женщин в постменопаузе с наличием в анамнезе ишемического инсульта или транзиторной ишемической атаки, терапию эстрогенами не следует назначать для вторичной профрилактики инсульта.

Влияние на свертывающую систему крови

(5) Венозная тромбоэмболия - одно из основных нежелательных явлений, отмечаемых на фроне применения пероральной ЗГТ и селективных модуляторов эстрогенных рецепторов. Риск возрастает с повышением дозы эстрогена, индекса массы тела с возрастом и выше в первые годы терапии.

(5) Применение 17 $\beta$-эстрадиола (но не этинилэстрадиола) в различных лекарственных формах, не предназначенных для перорального приема, может быть предпочтительней при повышенном риске венозной тромбоэмболии благодаря отсутствию эффректа первичного прохождения препарата через печень.

\section{Болезнь Альцгеймера}

По данным наблюдений, применение ЗГТ у женщин более молодого возраста в период менопаузы связано с более низким риском развития болезни Альцгеймера.

\section{Онкологические риски}

\section{ЗГТ и рак молочной железы}

(3 Результаты исследования WHI показали, что в группе применения эстрогенов с прогестинами повышалась частота РМЖ в среднем после 5,6 лет наблюдения, однако после поправки на другие факторы эта величина оказалась статистически незначимой. Женщины, которым не проводили ЗГТ до исследования, не имели более высокого риска РМЖ в течение 7 лет после начала терапии.

(5) Применение микронизированного прогестерона или дидрогестерона в сочетании с пероральным или трансдермальным эстрадиолом имеет лучший профиль в отношении риска развития РМЖ по сравнению с синтетическими прогестагенами как минимум в течение 5 лет. Однако глубокие клинические исследования в этом направлении пока не проводились.

(3 Риск диагностирования РМЖ резко снижается после прекращения ЗГТ; через 5 лет он может быть не выше, чем у женщин, которые не применяли такое лечение.

5 Факторы образа жизни, повышающие риск РМЖ, включают постменопаузальное ожирение, повышенное потребление алкоголя, сниженную фризическую активность.

( $\mathrm{K}$ фракторам, на которые невозможно повлиять, относятся семейный анамнез, повышенная маммографрическая плотность груди, атипичная гиперплазия протоков. 
Повышенный риск РМЖ, отмечаемый при применении ЗГТ, можно отчасти снизить путем отбора для женщин терапии с меньшим индивидуальным исходным риском и предоставления информации о профилактических мерах, направленных на поддержание здорового образа жизни.

\section{ЗГТ и эндометрий}

- Монотерапия эстрогенами сопровождается повышением риска развития гиперплазии и рака эндометрия, который зависит от дозы и длительности терапии. Этот повышенный риск сохраняется в течение многих лет после прекращения лечения.

(5 Прогестагены препятствуют пролифрерации эндометрия, вызванной эстрогенами.

Для защиты эндометрия необходимо применять прогестаген в соответствующей дозе в течение не менее 12 из 30 дней.

( Непрерывный режим комбинированной терапии связан с более низким риском развития рака эндометрия по сравнению с популяцией без лечения.

与 Новые режимы терапии с применением низких доз вызывают меньшую стимуляцию эндометрия и меньше кровотечений.

( Применение ЗГТ обычно не рекомендуется после лечения рака эндометрия, хотя данных пока недостаточно.

Ожирение повышает риск развития патологии эндометрия.

\section{ЗГТ и рак яичников}

( Исследование WHI - единственное рандомизированное клиническое исследование, в котором изучали риск развития рака яичников при ЗГТ. У женщин, принимавших комбинированную ЗГТ, не наблюдалось значительного повышения такого риска.

В целом длительная монотерапия эстрогенами может быть связана с небольшим относительным риском развития рака яичников, составляющим 0,7 случая на 1000 женщин за 5 лет применения, в то время как при использовании комбинации эстроген + прогестаген риск значительно ниже либо вообще отсутствует.

\section{ЗГТ и колоректальный рак}

Большинство обсервационных исследований показали снижение риска развития колоректального рака у женщин на фроне применения пероральной ЗГТ.

- Результаты трех метаанализов свидетельствуют о снижении риска колоректального рака при ЗГТ и сохранении благоприятного эфффекта в течение 4 лет после прекращения терапии.

\section{ЗГТ и рак шейки матки}

Длительные когортные исследования показали отсутствие повышенного риска развития рака шейки матки при применении ЗГТ.

5 В исследовании WHI на фоне ЗГТ повышения риска рака шейки матки не наблюдалось.

\section{Дополнительные методы лечения вазомоторных симптомов}

На данный момент в высококачественных исследованиях не было получено согласованных доказательств эфрфективности вспомогательных или безрецептурных препаратов в снижении тяжести и частоты приливов или ночной потливости.
«Биоидентичные» или «натуральные» гормоны

9 Такие названия, как «биоидентичные» или «натуральные» гормоны и их реклама не имеют научного обоснования, позволяющего отделить их от множества зарегистрированных препаратов для ЗГТ.

5 Так называемые натуральные, но синтезированные человеком гормоны могут содержаться в «биоидентичных» препаратах в комбинациях и дозах, которые никогда не изучались в публичных качественных клинических исследованиях. Данные об эфрфективности и безопасности длительной терапии любым из этих препаратов отсутствуют.

( С длительным применением эстрогенсодержащих биоидентичных гормонов может быть связано развитие патологии эндометрия.

\section{Постменопаузальная вагинальная атрофия}

Вагинальная атрофия становится клинически выраженной через 4-5 лет после наступления менопаузы, и объективные изменения, так же как и субъективные жалобы, отмечаются у 25-50\% всех женщин в постменопаузе.

Следует как можно раньше выявлять постменопаузальную атрофию и назначать соответствующее лечение на ранней стадии, прежде чем произойдут необратимые атрофические изменения.

Л Лечение необходимо продолжать для сохранения положительного эффекта.

Все местные препараты эстрогенов эффрективны, и выбор метода лечения обычно зависит от предпочтений пациентки.

(5анные о применении вагинальных эстрогенов у женщин с гинекологическими видами гормональнозависимого рака ограничены, и, следовательно, при их применении необходимо соблюдать осторожность.

Таким образом, принимая во внимание обновленные рекомендации IMS (2011) по применению ЗГТ, можно отметить несколько важных пунктов, а именно:

Применение ЗГТ очень актуально и является частью общей стратегии сохранения здоровья и качества жизни в зрелом возрасте.

3 Требуются дальнейшие многоцентровые масштабные испытания по исследованию влияния ЗГТ на организм в целом с целью минимизации возможных рисков ее применения.

5 Необходим индивидуальный подход в выборе препаратов, режимов, доз и пути введения в каждом конкретном случае с вычислением конкретных рисков и ожидаемой пользы у каждой отдельной женщины, принимая во внимание возраст и преморбидный фон.

Итак, на сегодняшний день в арсенале современного врача имеется широкий ассортимент препаратов ЗГТ, что позволит ему, используя огромную базу данных доказательной медицины, не только выполнять свою главную миссию - лечение, но и осуществлять давно забытое предназначение медицины - пропагандировать современные медицинские знания среди коллег и населения. 